\title{
Cytotoxic Effects of 27 Anticancer Drugs in HeLa and MDR1-Overexpressing Derivative Cell Lines
}

\author{
Kohji Takara, ${ }^{* a}$ Toshiyuki Sakaeda, ${ }^{b}$ Tatsurou Yagami, ${ }^{c}$ Hironao Kobayashi, ${ }^{c}$ Nobuko Ohmoto, ${ }^{b}$ \\ Masanori Horinouchi, ${ }^{b}$ Kohshi Nishiguchi, ${ }^{b}$ and Katsuhiko OKUMURA ${ }^{b}$ \\ ${ }^{a}$ Department of Hospital Pharmacy, Faculty of Pharmaceutical Sciences, Kyoto Pharmaceutical University; 5 Nakauchi- \\ cho, Misasagi, Yamashina-ku, Kyoto 607-8414, Japan: ${ }^{b}$ Department of Hospital Pharmacy, School of Medicine, Kobe \\ University; 7-5-2 Kusunoki-cho, Chuo-ku, Kobe 650-0017, Japan: and ${ }^{c}$ Shionogi Research Laboratories, Shionogi \& Co., \\ Ltd.; 5-12-4 Sagisu, Fukushima-ku, Osaka 553-0002, Japan. \\ Received December 12, 2001; accepted March 11, 2002
}

The cytotoxic effects of 27 anticancer drugs including amrubicin, vinorelbine, paclitaxel, docetaxel, gemcitabine, and irinotecan were evaluated in human cervical carcinoma HeLa cells, and drug-resistant HeLa-derived Hvr1-1, Hvr10-6, and Hvr100-6 cells, which were newly established by stepwise exposure to vinblastine. FACS and RT-PCR analysis indicated that MDR1 (P-glycoprotein) was induced without any alterations in expression of its related transporters. Hvr100-6 cells showed 2- to 200-fold higher resistance to anthracyclines than HeLa cells, and unexpectedly showed slight resistance to idarubicin and amrubicin. The relative resistance to vinca-alkaloids was 300- to 600000 -fold, and Hvr100-6 cells showed the highest relative resistance to vinorelbine. Hvr100-6 cells also showed 4000- and 60000-fold resistance to the taxanes paclitaxel and docetaxel, respectively. Hvr100-6 cells were also resistant to 6-mercaptopurine, actinomycin $D$, etoposide, and mitomycin $C$, with relative resistance of 8-, 45000-, 12-, and 9-fold, respectively. In contrast, Hvr100-6 cells showed no or slight resistance to platinum derivatives, pyrimidine analogues, and alkylating agents or to irinotecan and its active form, or tamoxifen. The cytotoxicity of anthracyclines, vinca-alkaloids, taxanes, actinomycin D, and etoposide was extensively reversed by cyclosporin A. Cyclosporin A had no effect on the cytotoxicity of 6-mercaptopurine or mitomycin $\mathrm{C}$, suggesting that resistance to these drugs was not mediated via MDR1. The alterations in cytotoxicity by overexpression of MDR1 and effects of cyclosporin A could be also qualitatively explained by $\left[^{3} \mathrm{H}\right]$ vinblastine uptake experiments. The 27 anticancer drugs analyzed here could be classified into substrates and nonsubstrates for MDR1. This will be useful for designing effective regimens for chemotherapy.

Key words MDR1; multidrug resistance; HeLa cell; cytotoxicity; anticancer drug

Combination chemotherapy using anticancer drugs with different modes of action is one of the most common forms of cancer treatment. A great deal of effort has been applied to the development of novel anticancer drugs and combination dosage regimens, which have provided effective cures for some previously lethal forms of malignancy. ${ }^{1)}$ These efforts have, however, been seriously hampered by the intrinsic or acquired resistance of a large number of cancers to anticancer drugs, since chemotherapy itself is sometimes responsible for the selection or induction of resistant clones. ${ }^{2,3)}$ The acquisition of multidrug resistance (MDR) of tumor cells still remains one of the major obstacles in cancer chemotherapy. ${ }^{4-6)}$

Recent developments in molecular pharmacology and cell biology have suggested that resistance may be due to failure of drug uptake or activation, ${ }^{7-10)}$ alterations in target enzymes including topoisomerase, ${ }^{11-13)}$ activation of enzymatic systems involved in the repair of damage to DNA, ${ }^{14}$ enhanced expression of detoxifying enzymes including gultathione-S-transferase, ${ }^{15-18)}$ or increased anticancer drug efflux. ${ }^{19-21)}$ The acquisition of the MDR phenotype against anticancer drugs in patients after chemotherapy has also been reported in acute myeloid leukemia, acute lymphoblastic leukemia, non-Hodgkin's lymphoma, and breast, gastrointestinal, gynecological, and genitourinary tumors., ${ }^{2,3}$ Clinically, MDR must be multifactorial, but is often discussed in reference to the expression of the anticancer drug efflux protein MDR1 (P-glycoprotein) encoded by the MDR1 gene, and its related proteins including multidrug resistance-associated protein 1 (MRP1), multidrug resistance-associated protein 2 (MRP2), and breast cancer resistance protein (BCRP). These efflux proteins actively expel structurally and functionally unrelated anticancer drugs from cells, decreasing their intracellular accumulation to noncytotoxic levels. ${ }^{720-23)}$ During the past decade, due to a correlation between MDR1 expression in patient tumor specimens and poor chemotherapy treatment prognosis, ${ }^{2,3)}$ the clinical relevance of MDR1 rather than other related ATP-binding cassette $(\mathrm{ABC})$ transporters has attracted a great deal of interest.

In the past few years, a number of novel anticancer drugs have been developed, including the inhibitor of topoisomerase II amrubicin hydrochloride (AMR), the inhibitor of microtubule depolymerization vinorelbine ditartrate (NVB), promoters of microtubule polymerization paclitaxel (TXL) and docetaxel (TXT), the antimetabolite gemcitabine hydrochloride (GEM), and the inhibitor of topoisomerase I irinotecan (CPT-11). The efficacies of novel combination chemotherapy regimens including these agents as key drugs have been examined in clinical trials.

In this study, three derivative cell lines were successively established from the human cervical carcinoma cell line HeLa-Ohio (HeLa) via stepwise exposure to vinblastine sulfate (VLB), and named Hvr1-1, Hvr10-6, and Hvr100-6 cells. The characteristics of Hvr1-1, Hvr10-6, and Hvr100-6 cells were first investigated using flow cytometric analysis, reverse transcription-polymerase chain reaction (RT-PCR), and growth inhibition assay. Using these cells, the cytotoxic effects of 27 anticancer drugs including AMR, NVB, TXL, TXT, GEM, and CPT-11 were examined. The effects of cyclosporin A (CyA), a typical substrate of MDR $1,{ }^{24,25)}$ on the 
cytotoxicity of these anticancer drugs were also assessed, and mechanisms underlying the alterations in cytotoxicity were examined by uptake experiments using $\left[{ }^{3} \mathrm{H}\right] \mathrm{VLB}$.

\section{MATERIAL AND METHODS}

Chemicals AMR was a kind gift from Sumitomo Pharmaceuticals Co., Ltd. (Osaka, Japan), TXT was from Aventis Pharma Ltd. (Vitry sur Seine, France), GEM was from Eli Lilly \& Co. (Indianapolis, IN, U.S.A.), idarubicin hydrochloride (IDR) was from Pharmacia \& Upjohn (Bridgewater, NJ, U.S.A.), CPT-11 and its active form SN-38 were from Daiichi Pharmaceutical Co., Ltd. (Tokyo, Japan), nedaplatin (254-S) and vindesine sulfate (VDS) were from Shionogi \& Co., Ltd. (Osaka, Japan), nimustine (ACNU) was from Sankyo Co., Ltd. (Tokyo, Japan), and NVB was from Pierre Fabre Medicament (Castres, France). Aclarubicin (ACR), actinomycin D (ACT-D), carboplatin (CBDCA), cyclophosphamide monohydrate (CPA), daunorubicin hydrochloride (DNR), doxorubicin hydrochloride (DXR), etoposide (VP16), 5-fluorouracil (5-FU), 6-mercaptopurine monohydrate (6-MP), mitomycin C (MMC), TXL, pirarubicin (THP), vinblastine sulfate (VLB), vincristine sulfate (VCR), and CyA were purchased from Wako Pure Chemical Industries, Ltd. (Osaka, Japan). Cytosine-1- $\beta$-D(+)-arabynofuranoside (AraC), melphalan (L-PAM), and tamoxifen citrate (TAM) was purchased from Sigma Chemical Co. (St. Louis, MO, U.S.A.). Cisplatin (CDDP) was purchased from Aldrich Chemical Co. (Milwaukee, WI, U.S.A.). ${ }^{3}$ H]VLB $(422$ $\mathrm{GBq} / \mathrm{mmol}$ ) was obtained from Amersham International, plc (Buckinghamshire, U.K.). Other chemicals used were of the highest purity available.

Cells and Cell Culture The human cervical carcinoma cell line HeLa was obtained from Dainippon Pharmaceutical Co., Ltd. (Osaka, Japan). Hvr1-1, Hvr10-6, and Hvr100-6 cells were newly established by stepwise exposure of HeLa cells to VLB. HeLa (396 - 401 passages), Hvr1-1 (34-38 passages), Hvr10-6 (107-113 passages), and Hvr100-6 (82-87 passages) cells were maintained in culture medium consisting of Dulbecco's modified Eagle's medium (D-MEM, cat. no. 12800-017, Invitrogen, Corp., Carlsbad, CA, U.S.A.) supplemented with $10 \%$ fetal bovine serum (FBS, lot no. AGM7413, HyClone, Utah, U.S.A.) and kanamycin sulfate $100 \mathrm{mg} / 1$ (Invitrogen). In the case of Hvr1-1, Hvr10-6, and Hvr100-6 cells, VLB was also added to the culture medium at final concentrations of 1,10 , and $100 \mathrm{nM}$, respectively. HeLa, Hvr1-1, Hvr10-6, and Hvr100-6 cells (4-12×10 cells $/ \mathrm{cm}^{2}$ ) were seeded into culture flasks (Nunclon flasks, Nalge Nunc International, U.S.A.), grown in a humidified atmosphere of $5 \% \mathrm{CO}_{2}-95 \%$ air at $37^{\circ} \mathrm{C}$, and were subcultured every 3 or $4 \mathrm{~d}$ with $0.02 \%$ EDTA and $0.05 \%$ trypsin (Invitrogen).

Assay for Cell Growth Activity The growth of Hvr1-1, Hvr10-6, and Hvr100-6 cells as well as HeLa cells was evaluated based on their growth curves. HeLa, Hvr1-1, Hvr10-6, and Hvr100-6 cells (1000 cells/well) were seeded on 96-well plates (Nalge Nunc International) on day 0 in $100 \mu \mathrm{l}$ of culture medium without VLB, and the cell number was counted every day from day 0 to day 6 . Cell number was evaluated by the WST-1 (tetrazolium salts) colorimetric assay using a Cell Counting Kit (Dojindo Laboratories, Kumamoto, Japan), based on the MTT assay. ${ }^{26-28)}$ According to the manufacturer's instructions, the culture medium was exchanged with $110 \mu \mathrm{l}$ of medium containing WST-1 reagent solution (WST$110 \mu \mathrm{l}+100 \mu \mathrm{l}$ of culture medium), and $3 \mathrm{~h}$ later the absorbance was determined at $450 \mathrm{~nm}$ with a reference wavelength of $630 \mathrm{~nm}$ using a microplate reader (Sjeia Auto Reader II; Sanko Junyaku Co. Ltd., Tokyo, Japan). Preliminary experiments demonstrated a good proportional relationship between the absorbance and the cell number (ranging from 500 to 32000 cells/well) in each cell line, with acceptable precision and accuracy and very small day-to-day or within-day variations. The doubling time of each cell line was calculated by the following eq. 1 .

doubling time $=\left(t-t_{0}\right) \times \log 2 /\left(\log N-\log N_{0}\right)$

where $t$ and $t_{0}$ represent the time of cell counting, and $N$ and $N_{0}$ represent the cell numbers at $t$ and $t_{0}$, respectively.

Detection of MDR1 and MRP1 by Flow Cytometry and RT-PCR Analysis of MDR1, MRP1, MRP2, and BCRP mRNA Expression HeLa, Hvr1-1, Hvr10-6, and Hvr100-6 cells were treated with EDTA and harvested in Dulbecco's modified phosphate-buffered saline without magnesium and calcium (PBS; $137.9 \mathrm{~mm} \mathrm{NaCl}, 8.1 \mathrm{~mm} \mathrm{Na} \mathrm{HPO}_{4}, 2.7 \mathrm{~mm}$ $\left.\mathrm{KCl}, 1.5 \mathrm{mM} \mathrm{KH}_{2} \mathrm{PO}_{4}\right)$. Each cell line $\left(1 \times 10^{6}\right.$ cells $)$ was incubated with the MDR1-specific monoclonal antibodies ${ }^{29}$ ) MRK-16 (1:5 dilution, Funakoshi Co., Ltd., Tokyo, Japan) and JSB-1 (1:10 dilution, Sanbio BV, Netherlands), or the MRP1-specific monoclonal antibody MRPm6 (1:20 dilution, Sanbio BV) for $60 \mathrm{~min}$ on ice. As a secondary antibody, fluorescence isothiocyanate-conjugated goat anti-mouse IgG (Becton Dickinson \& Co., NJ, U.S.A.) was used, and the cells were incubated for $30 \mathrm{~min}$ on ice. The cells were washed twice with PBS, and the fluorescence intensity of aliquots of $1 \times 10^{5}$ cells was determined using a fluorescence-activated cell sorter $\left(\right.$ FACScan $^{\mathrm{TM}}$, Becton Dickinson \& Co.).

The expression of MDR1, MRP1, MRP2, and BCRP mRNA in HeLa and Hvr100-6 cells was measured by the RTPCR method..$^{30,31)}$ Total RNA was isolated using the RNeasy $^{\circledR}$ mini kit (Qiagen Inc., CA, U.S.A.), and aliquots $(0.3 \mu \mathrm{g})$ of RNA were used for reverse transcription and cDNA-PCR, using an RNA PCR kit (AMV) ver. 2.1 (Takara Shuzo Co., Ltd., Shiga, Japan). The PCR primer sequences of MDR1, MRP1, MRP2, BCRP, and $\beta 2$-microglobulin $(\beta 2 \mathrm{~m})$ used as an internal control were as follows: MDR1 sense primer 5'-CCC ATC ATT GCA ATA GCA GG-3' and antisense primer $5^{\prime}$-GTT CAA ACT TCT GCT CCT GA-3' corresponding to $167-\mathrm{bp}$; MRP1 sense primer 5'-ATC AAG ACC GCT GTC ATT GG-3' and antisense primer 5'-GAG CAA GGA TGA CTT GCA GG-3' corresponding to 181-bp; MRP2 sense primer 5'-CTG CCT CTT CAG AAT CTT AG$3^{\prime}$ and antisense primer 5'-CCC AAG TTG CAG GCT GGC C-3' corresponding to 241-bp; BCRP sense primer 5' -TGC CCA GGA CTC AAT GCA ACA G-3' and antisense primer 5'-ACA ATT TCA GGT AGG CAA TTG TG-3' corresponding to $172-\mathrm{bp} ; \beta 2 \mathrm{~m}$ sense primer $5^{\prime}$-ACC CCC ACT GAA AAA GAT GA-3' and antisense primer 5'-ATC TTC AAA CCT CCA TGA TG-3' corresponding to 120-bp. PCR amplification was initiated by one cycle at $94{ }^{\circ} \mathrm{C}$ for $2 \mathrm{~min}$ followed by 30 sequential cycles of denaturation at $94^{\circ} \mathrm{C}$ for $1 \mathrm{~min}$, annealing at $60^{\circ} \mathrm{C}$ for $1 \mathrm{~min}$, and extension at $72^{\circ} \mathrm{C}$ for $1 \mathrm{~min}$ in a thermal cycler (iCycler Thermal Cycler, Bio- 
Rad Laboratories, Inc., CA, U.S.A.). PCR products were separated on Tris-acetate-EDTA 3\% agarose gels containing ethidium bromide $100 \mathrm{ng} / \mathrm{ml}$. Densitometric analysis was performed on a Macintosh computer using the NIH Image program ver. 1.62 (National Institutes of Health, Bethesda, MD, U.S.A.).

Growth Inhibition Assay The cytotoxicity of anticancer drugs in the cells was also evaluated using the WST-1 colorimetric assay. Each cell line (1000 cells/well) was seeded on 96-well plates (Nalge Nunc International) in $100 \mu$ l of culture medium without anticancer drugs on day 0 , and $24 \mathrm{~h}$ later the culture medium was exchanged with that containing the test anticancer drug at various concentrations (on day 1). The anticancer drugs were added using ethanol, dimethyl sulfoxide, or double-distilled water. The maximum concentration of organic solvents or double-distilled water was $1 \%$, which had no effect on cell viability or growth. After incubation for $3 \mathrm{~d}$ at $37^{\circ} \mathrm{C}$, the WST- 1 colorimetric assay was performed as described above (on day 4).

The $50 \%$ growth inhibitory concentration $\left(\mathrm{IC}_{50}\right)$ of the anticancer drugs in HeLa, Hvr1-1, Hvr10-6, and Hvr100-6 cells was calculated according to the sigmoid inhibitory effect model as shown in equation (2) by means of a nonlinear least-squares fitting method (WinNonlin ${ }^{\circledR}$, ver. 2.1, Pharsight Corp., CA, U.S.A.):

$$
E=E_{\max } \times\left[1-C^{\gamma} /\left(C^{\gamma}+\mathrm{IC}_{50}{ }^{\gamma}\right)\right]
$$

where $E$ and $E_{\max }$ represent the surviving fraction (\% of control) and its maximum, respectively, and $C$ and $\gamma$ represent the drug concentration in the medium ( $\mathrm{pm}, \mathrm{nM}$, or $\mu \mathrm{M})$ and the sigmoidicity factor, respectively. The relative resistance to anticancer drugs of Hvr1-1, Hvr10-6, and Hvr100-6 cells compared with HeLa cells was defined as the ratio of their $\mathrm{IC}_{50}$ values. Each series of experiments was conducted in quadruplicate, and values are presented here as means \pm S.D. after rounding off to three significant figures.

Assay for MDR Reversal by CyA To confirm the contribution of MDR1 to MDR phenotypes of Hvr100-6 cells, the effects of CyA, a representative substrate for MDR $1,{ }^{24,25)}$ on the cytotoxicity of anticancer drugs were evaluated using the WST-1 assay. Cells were seeded on day 0 , and the culture medium was exchanged with that containing CyA ( 1 or $5 \mu \mathrm{M})$ together with each test anticancer drug on day 1 , followed by the same procedures as described above. The maximum concentration of CyA that had no effect on cell viability or growth was $5 \mu \mathrm{M}$.

[ ${ }^{3}$ H]VLB Uptake Assay The cells $\left(2 \times 10^{5}\right.$ cells/well $)$ were seeded on 24-well plates (Nalge Nunc International) in the culture medium without VLB, and cultured for $2 \mathrm{~d}$ in a humidified atmosphere of $5 \% \mathrm{CO}_{2}-95 \%$ air at $37^{\circ} \mathrm{C}$. The cells were then washed twice with warmed complete Hank's balanced salt solution (HBSS, cat. no. H-6136, Sigma Chemical), and the uptake experiments were immediately started by the addition of HBSS including $\left[{ }^{3} \mathrm{H}\right] \mathrm{VLB}(1 \mu \mathrm{M}, 37 \mathrm{kBq})$ with or without CyA (1 or $5 \mu \mathrm{M})$ to each well, followed by incubation at $37^{\circ} \mathrm{C}$. The experiment was stopped by aspiration of HBSS from each well $2 \mathrm{~h}$ later. The cells were then quickly washed three times with ice-cold PBS on ice, and subsequently lysed in $0.3 \mathrm{~N} \mathrm{NaOH} 1 \mathrm{ml}$. Radioactivity of the lysed cells was counted in $3 \mathrm{ml}$ of ACS II (Amersham International) by liquid scintillation counting (LS6000TA, Beck- man Instruments, Inc., CA, U.S.A.). The radioactivity in the washing solution was also determined in each experiment, and was usually around the background level.

Protein Assay Protein content was determined using a Bio-Rad Protein Assay kit (Bio-Rad Laboratories, CA, U.S.A.). Bovine $\gamma$-globulin was used as the standard.

Statistical Analysis Multiple comparisons were performed by one-way analysis of variance (ANOVA) followed by the Scheffé F-test. $p$ values of less than 0.05 (two-tailed) were considered significant.

\section{RESULTS}

Growth Curves of HeLa, Hvr1-1, Hvr10-6, and Hvr100-6 Cells Growth activity was evaluated in Hvr1-1, Hvr10-6, and Hvr100-6 cells as well as HeLa cells. In each growth curve, the lag phase of cell growth was within $1 \mathrm{~d}$ after cell seeding, and then the logarithmic phase continued for at least $6 \mathrm{~d}$ (Fig. 1). The growth activity of Hvr1-1, Hvr10-6, and Hvr100-6 cells was comparable or slightly decreased as compared with that of HeLa cells, with cell doubling times of 23, 28, 31, and $23 \mathrm{~h}$, respectively (Table 1).

Expression of MDR1, MRP1, MRP2, and BCRP in HeLa, Hvr1-1, Hvr10-6, and Hvr100-6 Cells FACS analysis was performed using MRK-16 and JSB-1 antibodies for MDR1 and MRPm6 antibody for MRP1 in HeLa, Hvr11, Hvr10-6, and Hvr100-6 cells (Fig. 2). Stepwise exposure of HeLa cells to VLB induced only MDR1 with no induction of MRP1. No shift in signal was seen with JSB-1 antibody. FACS analysis of Hvr1-1 and Hvr10-6 cells indicated that this induction occurred in succession in the order Hvr1-1, Hvr10-6, and Hvr100-6 cells (data not shown). RT-PCR analysis also indicated that the expression of MDR1 mRNA in Hvr100-6 cells markedly higher than that in HeLa cells, and that of MRP2 was slightly higher than that in HeLa cells. The expression levels of MRP1 and BCRP mRNA in Hvr100-6 cells were comparable to those in HeLa cells (data not shown).

Cytotoxicity of Anticancer Drugs in HeLa, Hvr1-1, Hvr10-6, and Hvr100-6 Cells The cytotoxic effects of VLB and 5-FU, the representative substrate and nonsubstrate for MDR1, respectively, on HeLa, Hvr1-1, Hvr10-6, and Hvr100-6 cells were evaluated using the WST-1 assay (Fig. 3 and Table 1). The growth inhibitory curves of Hvr1-1, Hvr10-6, and Hvr100-6 cells by VLB shifted in that order to

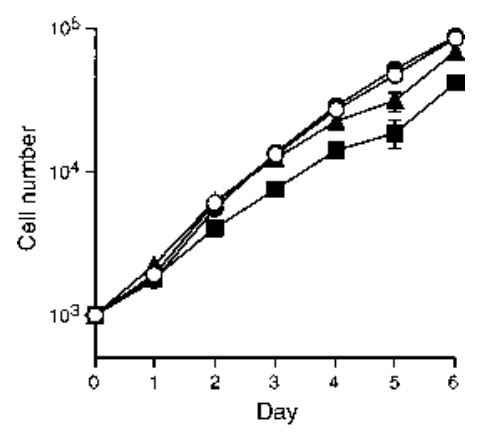

Fig. 1. Growth Curves for HeLa, Hvr1-1, Hvr10-6, and Hvr100-6 Cells Each cell line was seeded at 1000 cells/well on 96-well plates on day 0 . Cell number was evaluated using the WST-1 assay (see Materials and Methods section) from day 0 to day 6. $\bigcirc$, HeLa cells;, Hvr1-1 cells; $\boldsymbol{\Delta}$, Hvr10-6 cells; $\boldsymbol{\square}$, Hvr100-6 cells. Each point represents the mean \pm S.D. of quadruplicate experiments. 


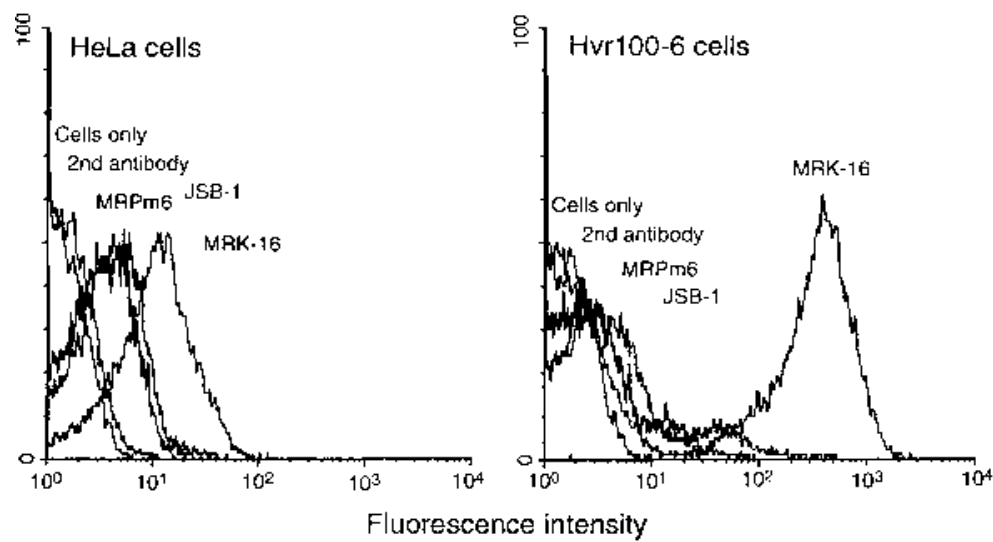

Fig. 2. Expression of MDR1 and MRP1 in HeLa (Left) and Hvr100-6 Cells (Right) by Flow Cytometry

MRK-16 and JSB-1 antibodies for MDR1, and MRPm6 antibody for MRP1 were used in FACS analysis. The signals obtained with cells only, second antibody only, and MRK16, JSB-1, and MRPm6 antibodies are indicated.

Table 1. Overview of HeLa, Hvr1-1, Hvr10-6, and Hvr100-6 Cell Characteristics

\begin{tabular}{|c|c|c|c|c|c|c|c|}
\hline \multirow{2}{*}{ Cell line } & \multirow{2}{*}{$\begin{array}{l}\text { Doubling } \\
\text { time (h) }\end{array}$} & \multicolumn{3}{|c|}{ Vinblastine } & \multicolumn{3}{|c|}{ 5-Fluorouracil } \\
\hline & & $\mathrm{IC}_{50}(\mathrm{nM})$ & $\gamma$ & $\mathrm{RR}^{a)}$ & $\mathrm{IC}_{50}(\mu \mathrm{M})$ & $\gamma$ & $\mathrm{RR}$ \\
\hline HeLa & 23.4 & $0.1 \pm 0.0$ & $1.6 \pm 0.4$ & - & $2.9 \pm 0.7$ & $0.9 \pm 0.1$ & - \\
\hline Hvr1-1 & 23.0 & $1.6 \pm 0.2$ & $1.8 \pm 0.4$ & 12 & $2.8 \pm 0.9$ & $0.6 \pm 0.0$ & 1.0 \\
\hline Hvr10-6 & 28.1 & $22.3 \pm 6.5$ & $2.3 \pm 0.6$ & 166 & $4.1 \pm 0.8$ & $0.5 \pm 0.0$ & 1.4 \\
\hline Hvr100-6 & 31.0 & $67.0 \pm 30.1$ & $1.3 \pm 0.5$ & 498 & $11.5 \pm 2.3$ & $0.7 \pm 0.1$ & 4.0 \\
\hline
\end{tabular}

a) The relative resistance $=\mathrm{IC}_{50}$ values in $\mathrm{Hvr} 100-6$ cells $/ \mathrm{IC}_{50}$ values in HeLa cells.
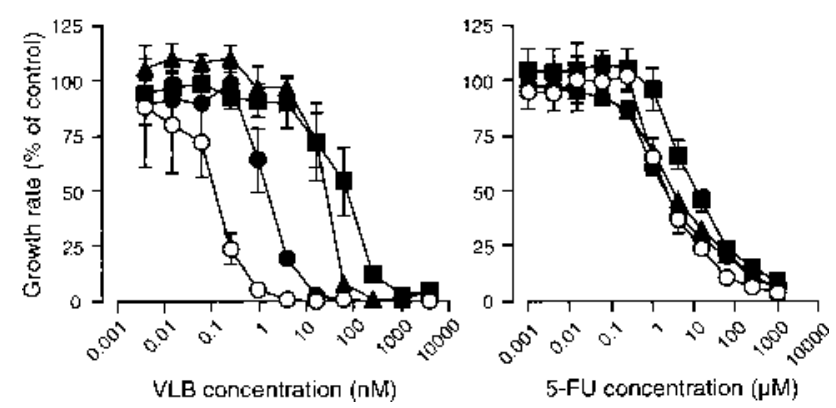

Fig. 3. Growth-Inhibitory Curves of HeLa, Hvr1-1, Hvr10-6, and Hvr1006 Cells by Vinblastine (Left Side) and 5-Fluorouracil (Right Side)

O, HeLa cells; ๑, Hvr1-1 cells; $\boldsymbol{\Lambda}$, Hvr10-6 cells; $\mathbf{\square}$, Hvr100-6 cells. Each point represents the mean \pm S.D. of quadruplicate experiments.

the higher ranges of concentration with no alterations in the slopes of the curves over the concentration range examined. Moreover, the growth inhibitory curves of HeLa, Hvr1-1, Hvr10-6, and Hvr100-6 cells by 5-FU were comparable, but Hvr100-6 cells showed very slight resistance ( $c a$. 4-fold).

The cytotoxic effects of 27 anticancer drugs on Hvr100-6 cells were compared with those on HeLa cells (Tables 2, 3). Hvr100-6 cells showed 2- to 200-fold higher resistance to anthracyclines than HeLa cells, and unexpectedly showed slight resistance (4-fold) to IDR and almost no resistance to AMR. Hvr100-6 cells were 300 - to 600000 -fold more resistant to vinca-alkaloids than HeLa cells, and Hvr100-6 cells showed the highest relative resistance to NVB. Hvr100-6 cells also showed 4000- and 60000-fold higher resistance to the taxanes TXL and TXT, respectively, than HeLa cells. Hvr100-6 cells were 45000-, 12-, and 9-fold more resistant to ACT-D,
VP-16, and MMC, respectively, than HeLa cells. Unexpectedly, these cells showed 8-fold higher resistance to 6-MP as compared with HeLa cells. In contrast, Hvr100-6 cells showed no or slight resistance to platinum derivatives, pyrimidine analogues and alkylating agents as well as to CPT-11 and its active form $\mathrm{SN}-38$, and TAM.

The $\gamma$ values in Hvr100-6 cells were similar to those in HeLa cells against all anticancer drugs, with the exception of DXR and DNR, for which the $\gamma$ values were decreased, and 6-MP and ACT-D, for which the $\gamma$ values increased.

Effects of CyA on Cytotoxicity of Anticancer Drugs in HeLa and Hvr100-6 Cells The effects of CyA, a typical substrate of MDR1, on the cytotoxic effects of anticancer drugs on Hvr100-6 cells were also evaluated (Tables 4, 5). The cytotoxicities of anthracyclines, vinca-alkaloids, taxanes, ACT-D, and VP-16, to which Hvr100-6 cells showed higher resistance, were extensively reversed by CyA, suggesting that the higher resistance of Hvr100-6 cells was mediated by MDR1. CyA had no effect on the cytotoxic effects of 6-MP or MMC, suggesting that the resistance to these drugs in Hvr100-6 cells was not mediated by MDR1.

Uptake of $\left[{ }^{3} \mathrm{H}\right]$ VLB in HeLa and Hvr100-6 Cells The uptake of $\left[{ }^{3} \mathrm{H}\right] \mathrm{VLB}$ over a period of $2 \mathrm{~h}$ was determined in Hvr100-6 as well as HeLa cells (Fig. 4). The uptake of $\left[{ }^{3} \mathrm{H}\right]$ VLB in Hvr100-6 cells was about 3-fold lower than in HeLa cells, and was increased by CyA in a concentration-dependent manner to a level comparable to that in HeLa cells.

\section{DISCUSSION}

Recently, another anticancer drug efflux protein, MRP1, has attracted attention as a prognostic indicator of poor effi- 
Table 2. $\mathrm{IC}_{50}$ Values for Anthracyclines, Vinca-Alkaloids, Taxanes, and Platinum Derivatives in HeLa and Hvr100-6 Cells

\begin{tabular}{|c|c|c|c|c|c|c|}
\hline \multirow{2}{*}{ Anticancer drug } & & \multicolumn{2}{|c|}{ HeLa cells } & \multicolumn{2}{|c|}{ Hvr100-6 cells } & \multirow{2}{*}{$\mathrm{RR}^{a)}$} \\
\hline & & $\mathrm{IC}_{50}$ & $\gamma$ & $\mathrm{IC}_{50}$ & $\gamma$ & \\
\hline \multicolumn{7}{|l|}{ Anthracyclines } \\
\hline Doxorubicin & $\mathrm{nm}$ & $2.94 \pm 0.535$ & $6.9 \pm 2.2$ & $153 \pm 56.8$ & $0.7 \pm 0.1$ & 52.1 \\
\hline Daunorubicin & nм & $1.09 \pm 0.309$ & $5.7 \pm 2.7$ & $216 \pm 87.3$ & $0.8 \pm 0.2$ & 198 \\
\hline Pirarubicin & $\mathrm{nm}$ & $0.108 \pm 0.0911$ & $0.4 \pm 0.0$ & $12.9 \pm 7.78$ & $0.5 \pm 0.1$ & 120 \\
\hline Aclarubicin & nM & $0.0200 \pm 0.0187$ & $0.3 \pm 0.1$ & $1.64 \pm 0.588$ & $0.5 \pm 0.0$ & 82.1 \\
\hline Idarubicin & nм & $1.74 \pm 0.375$ & $0.8 \pm 0.2$ & $6.70 \pm 2.30$ & $0.7 \pm 0.0$ & 3.84 \\
\hline Amrubicin & $\mathrm{nm}$ & $393 \pm 71.8$ & $1.6 \pm 0.4$ & $659 \pm 54.4$ & $1.6 \pm 0.1$ & 1.68 \\
\hline \multicolumn{7}{|l|}{ Vinca-alkaloids } \\
\hline Vinblastine & $\mathrm{nm}$ & $0.135 \pm 0.036$ & $1.6 \pm 0.4$ & $67.0 \pm 30.1$ & $1.3 \pm 0.5$ & 496 \\
\hline Vincristine & $\mathrm{nm}$ & $0.597 \pm 0.335$ & $1.5 \pm 0.1$ & $196 \pm 118$ & $1.0 \pm 0.2$ & 327 \\
\hline Vindesine & nм & $0.193 \pm 0.144$ & $0.7 \pm 0.1$ & $234 \pm 68.2$ & $1.6 \pm 0.5$ & 1214 \\
\hline Vinorelbine & $\mathrm{nm}$ & $0.000612 \pm 0.000372$ & $0.5 \pm 0.2$ & $376 \pm 130$ & $1.4 \pm 0.2$ & 614544 \\
\hline \multicolumn{7}{|l|}{ Taxanes } \\
\hline Paclitaxel & $\mathrm{nm}$ & $0.136 \pm 0.238$ & $0.7 \pm 0.7$ & $562 \pm 255$ & $1.1 \pm 0.3$ & 4145 \\
\hline Docetaxel & pм & $0.828 \pm 0.0772$ & $1.7 \pm 0.2$ & $47600 \pm 2600$ & $0.8 \pm 0.1$ & 57488 \\
\hline \multicolumn{7}{|c|}{ Platinum derivatives } \\
\hline Cisplatin & $\mu \mathrm{M}$ & $0.574 \pm 0.112$ & $0.9 \pm 0.1$ & $0.223 \pm 0.0458$ & $0.7 \pm 0.0$ & 0.389 \\
\hline Carboplatin & $\mu_{\mathrm{M}}$ & $10.0 \pm 2.67$ & $0.8 \pm 0.1$ & $8.17 \pm 2.32$ & $1.0 \pm 0.1$ & 0.817 \\
\hline Nedaplatin & $\mu \mathrm{M}$ & $1.76 \pm 0.661$ & $1.2 \pm 0.2$ & $1.10 \pm 0.0383$ & $0.9 \pm 0.2$ & 0.625 \\
\hline
\end{tabular}

The $\mathrm{IC}_{50}$ and $\gamma$ values represent the mean \pm S.D. of quadruplicate experiments. $a$ ) The relative resistance $=\mathrm{IC}_{50}$ values in $\mathrm{Hvr} 100-6$ cells $/ \mathrm{IC}_{50}$ values in HeLa cells.

Table 3. $\mathrm{IC}_{50}$ Values for Pyrimidine and Purine Analogues, Alkylating Agents, and Others in HeLa and Hvr100-6 Cells

\begin{tabular}{|c|c|c|c|c|c|c|}
\hline \multirow{2}{*}{ Anticancer drug } & & \multicolumn{2}{|c|}{ HeLa cells } & \multicolumn{2}{|c|}{ Hvr100-6 cells } & \multirow{2}{*}{$\mathrm{RR}^{a)}$} \\
\hline & & $\mathrm{IC}_{50}$ & $\gamma$ & $\mathrm{IC}_{50}$ & $\gamma$ & \\
\hline \multicolumn{7}{|l|}{ Pyrimidine analogues } \\
\hline 5-Fluorouracil & $\mu \mathrm{M}$ & $2.85 \pm 0.747$ & $0.9 \pm 0.1$ & $11.5 \pm 2.33$ & $0.7 \pm 0.1$ & 4.02 \\
\hline Cytarabine & $\mu \mathrm{M}$ & $0.198 \pm 0.0266$ & $0.6 \pm 0.1$ & $0.301 \pm 0.140$ & $0.4 \pm 0.1$ & 1.52 \\
\hline Gemcitabine & $\mu \mathrm{M}$ & $0.0149 \pm 0.00428$ & $1.4 \pm 0.3$ & $0.0299 \pm 0.0111$ & $0.7 \pm 0.2$ & 2.01 \\
\hline \multicolumn{7}{|l|}{ Purine analogue } \\
\hline 6-Mercaptopurine & $\mu \mathrm{M}$ & $16.7 \pm 4.89$ & $0.4 \pm 0.0$ & $133 \pm 23.1$ & $2.0 \pm 0.2$ & 7.97 \\
\hline \multicolumn{7}{|l|}{ Alkylating agents } \\
\hline Nimustine & $\mu \mathrm{M}$ & $6.07 \pm 2.85$ & $1.0 \pm 0.2$ & $11.3 \pm 10.3$ & $0.7 \pm 0.1$ & 1.86 \\
\hline Melphalan & $\mu \mathrm{M}$ & $2.69 \pm 0.323$ & $0.9 \pm 0.0$ & $4.74 \pm 0.313$ & $0.8 \pm 0.0$ & 1.76 \\
\hline Cyclophosphamide & $\mu_{\mathrm{M}}$ & $1689 \pm 247$ & $1.3 \pm 0.2$ & $1388 \pm 378$ & $1.3 \pm 0.3$ & 0.822 \\
\hline \multicolumn{7}{|l|}{ Others } \\
\hline Actinomycin D & nм & $0.000266 \pm 0.000222$ & $0.2 \pm 0.0$ & $12.0 \pm 1.39$ & $0.7 \pm 0.2$ & 44991 \\
\hline Etoposide & $\mu_{\mathrm{M}}$ & $0.234 \pm 0.0172$ & $1.5 \pm 0.9$ & $2.69 \pm 0.817$ & $0.9 \pm 0.2$ & 11.5 \\
\hline Irinotecan & $\mu \mathrm{M}$ & $1.82 \pm 1.02$ & $1.2 \pm 0.6$ & $6.12 \pm 2.69$ & $1.4 \pm 0.8$ & 3.36 \\
\hline $\mathrm{SN}-38$ & nм & $30.0 \pm 4.59$ & $0.9 \pm 0.1$ & $28.3 \pm 15.7$ & $0.4 \pm 0.1$ & 0.943 \\
\hline Mitomycin C & $\mu_{\mathrm{M}}$ & $0.0209 \pm 0.00576$ & $0.6 \pm 0.2$ & $0.187 \pm 0.0132$ & $0.8 \pm 0.0$ & 8.97 \\
\hline Tamoxifen & $\mu \mathrm{M}$ & $8.82 \pm 1.85$ & $8.6 \pm 2.6$ & $9.35 \pm 0.144$ & $9.9 \pm 0.0$ & 1.06 \\
\hline
\end{tabular}

The $\mathrm{IC}_{50}$ and $\gamma$ values represent the mean \pm S.D. of quadruplicate experiments. $a$ ) The relative resistance $=\mathrm{IC}_{50}$ values in $\mathrm{Hvr} 100-6$ cells $/ \mathrm{IC}_{50}$ values in HeLa cells.

cacy of chemotherapy. ${ }^{22,23)}$ These clinical observations necessitate separate evaluation of the contributions of MDR1 and its related proteins including MRP1, MRP2, and BCRP to MDR. Therefore we attempted to establish MDR1-induced tumor cell lines in which only MDR1 expression was up-regulated; Hvr1-1, Hvr10-6, and Hvr100-6 cells were established by stepwise exposure of HeLa cells to VLB. FACS and RT-PCR analysis demonstrated that MDR1 was successively induced without any induction of its related proteins including MRP1, MRP2, and BCRP.

In comparison with HeLa cells, Hvr100-6 cells showed resistance to anthracyclines, vinca-alkaloids, and taxanes, with relative resistance values of 2- to 200-, 300- to 600000-, and 4000- to 60000-fold, respectively. The resistance of Hvr1006 cells to these drugs was reversed by CyA in a concentra- tion-dependent manner. The cytotoxic effects of the anthracyclines IDR and AMR in Hvr100-6 cells were unexpectedly comparable to those in HeLa cells. Hvr100-6 cells showed a higher level of resistance to NVB than the other vinca-alkaloids, VLB, VCR, and VDS. IDR and AMR lack only the methoxy moiety of the anthraquinone ring as compared with DXR, DNR, and THP. NVB has only a slightly different chemical structure from VLB, VCR, and VDS. Moreover, the rank order of $\mathrm{Clog} P$ (the calculated logarithmic value of $n$-octanol/water partition coefficient, calculated using the computer program "CLOGP3" in "MedChem Softwave Release 3.54 Programs 1989," Chemical Information System Inc., U.S.A.) in anthracyclines was as follows: AMR $(\mathrm{C} \log \mathrm{P}=-0.78)$, DXR $(-0.50), \mathrm{DNR}(0.026)$, IDR (0.090), THP (0.94), and ACR (2.59), and those in vinca-alkaloids 
Table 4. Effects of Cyclosporin A on the Cytotoxicity of Anthracyclines, Vinca-Alkaloids, Taxanes, and Platinum Derivatives in Hvr100-6 Cells

\begin{tabular}{|c|c|c|c|c|c|c|c|}
\hline \multirow{2}{*}{ Anticancer drug } & & \multicolumn{3}{|c|}{$+\mathrm{CyA} 1 \mu \mathrm{M}$} & \multicolumn{3}{|c|}{+ CyA $5 \mu \mathrm{M}$} \\
\hline & & $\mathrm{IC}_{50}$ & $\gamma$ & $\mathrm{RR}^{a)}$ & $\mathrm{IC}_{50}$ & $\gamma$ & RR \\
\hline \multicolumn{8}{|l|}{ Anthracyclines } \\
\hline Doxorubicin & $\mathrm{nM}$ & $56.9 \pm 23.1$ & $0.6 \pm 0.1$ & 19.4 & $3.21 \pm 0.645$ & $0.8 \pm 0.1$ & 1.09 \\
\hline Daunorubicin & $\mathrm{nm}$ & $56.7 \pm 21.3$ & $0.6 \pm 0.1$ & 52.0 & $6.33 \pm 1.88$ & $1.0 \pm 0.2$ & 5.80 \\
\hline Pirarubicin & $\mathrm{nM}$ & $5.37 \pm 2.51$ & $0.4 \pm 0.0$ & 49.9 & $3.08 \pm 0.502$ & $0.8 \pm 0.1$ & 28.6 \\
\hline Aclarubicin & $\mathrm{nM}$ & $0.748 \pm 0.472$ & $0.4 \pm 0.1$ & 37.4 & $0.310 \pm 0.151$ & $0.5 \pm 0.0$ & 15.5 \\
\hline Idarubicin & $\mathrm{nM}$ & $2.43 \pm 1.87$ & $0.8 \pm 0.1$ & 1.39 & $3.09 \pm 0.601$ & $0.9 \pm 0.1$ & 1.77 \\
\hline Amrubicin & $\mathrm{nm}$ & $624 \pm 326$ & $1.4 \pm 0.6$ & 1.59 & $655 \pm 37.6$ & $1.9 \pm 0.2$ & 1.67 \\
\hline \multicolumn{8}{|l|}{ Vinca-alkaloids } \\
\hline Vinblastine & $\mathrm{nM}$ & $12.4 \pm 5.51$ & $0.6 \pm 0.1$ & 53.1 & $1.21 \pm 0.0708$ & $1.8 \pm 0.2$ & 5.18 \\
\hline Vincristine & $\mathrm{nM}$ & $102 \pm 17.3$ & $0.7 \pm 0.1$ & 171 & $1.32 \pm 0.126$ & $2.4 \pm 0.2$ & 2.21 \\
\hline Vindesine & $\mathrm{nm}$ & $20.8 \pm 13.7$ & $0.6 \pm 0.1$ & 108 & $0.743 \pm 0.181$ & $1.5 \pm 0.3$ & 3.85 \\
\hline Vinorelbine & $\mathrm{nm}$ & $57.9 \pm 28.4$ & $0.5 \pm 0.1$ & 94615 & $1.04 \pm 0.267$ & $1.4 \pm 0.4$ & 1704 \\
\hline \multicolumn{8}{|l|}{ Taxanes } \\
\hline Paclitaxel & $\mathrm{nM}$ & $58.5 \pm 18.3$ & $0.5 \pm 0.1$ & 431 & $1.59 \pm 0.267$ & $1.6 \pm 0.4$ & 11.7 \\
\hline Docetaxel & $\mathrm{pM}$ & $16200 \pm 9830$ & $0.5 \pm 0.1$ & 19618 & $182 \pm 33.7$ & $1.2 \pm 0.1$ & 220 \\
\hline \multicolumn{8}{|c|}{ Platinum derivatives } \\
\hline Cisplatin & $\mu \mathrm{M}$ & $0.158 \pm 0.0282$ & $0.7 \pm 0.0$ & 0.275 & $0.154 \pm 0.0926$ & $0.6 \pm 0.1$ & 0.268 \\
\hline Carboplatin & $\mu \mathrm{M}$ & $2.79 \pm 1.72$ & $0.9 \pm 0.3$ & 0.278 & $5.25 \pm 1.83$ & $1.2 \pm 0.1$ & 0.523 \\
\hline Nedaplatin & $\mu \mathrm{M}$ & $0.454 \pm 0.102$ & $0.9 \pm 0.1$ & 0.257 & $0.920 \pm 0.185$ & $0.9 \pm 0.1$ & 0.522 \\
\hline
\end{tabular}

The $\mathrm{IC}_{50}$ and $\gamma$ values represent the mean \pm S.D. of quadruplicate experiments. $a$ ) The relative resistance $=\mathrm{IC}_{50}$ values in $\mathrm{Hvr} 100-6$ cells $/ \mathrm{IC}_{50}$ values in $\mathrm{HeLa}$ cells.

Table 5. Effects of Cyclosporin A on the Cytotoxicity of Pyrimidine and Purine Analogues, Alkylating Agents, and Others in Hvr100-6 Cells

\begin{tabular}{|c|c|c|c|c|c|c|c|}
\hline \multirow{2}{*}{ Anticancer drug } & & \multicolumn{3}{|c|}{$+\mathrm{CyA} 1 \mu \mathrm{M}$} & \multicolumn{3}{|c|}{+ CyA $5 \mu \mathrm{M}$} \\
\hline & & $\mathrm{IC}_{50}$ & $\gamma$ & $\mathrm{RR}^{a)}$ & $\mathrm{IC}_{50}$ & $\gamma$ & $\mathrm{RR}$ \\
\hline \multicolumn{8}{|l|}{ Pyrimidine analogues } \\
\hline 5-Fluorouracil & $\mu_{\mathrm{M}}$ & $10.1 \pm 2.31$ & $0.5 \pm 0.0$ & 3.55 & $11.5 \pm 4.76$ & $0.6 \pm 0.0$ & 4.05 \\
\hline Cytarabine & $\mu_{\mathrm{M}}$ & $0.100 \pm 0.0398$ & $0.5 \pm 0.1$ & 0.505 & $0.171 \pm 0.106$ & $0.6 \pm 0.3$ & 0.868 \\
\hline Gemcitabine & $\mu \mathrm{M}$ & $0.0242 \pm 0.00707$ & $0.6 \pm 0.1$ & 1.62 & $0.0487 \pm 0.0132$ & $0.5 \pm 0.1$ & 3.27 \\
\hline \multicolumn{8}{|l|}{ Purine analogue } \\
\hline 6-Mercaptopurine & $\mu \mathrm{M}$ & $106 \pm 45.9$ & $1.5 \pm 0.7$ & 6.33 & $189 \pm 30.9$ & $3.4 \pm 2.6$ & 11.4 \\
\hline \multicolumn{8}{|l|}{ Alkylating agents } \\
\hline Nimustine & $\mu \mathrm{M}$ & $44.4 \pm 7.96$ & $0.6 \pm 0.1$ & 7.32 & $58.3 \pm 22.3$ & $0.6 \pm 0.1$ & 9.60 \\
\hline Melphalan & $\mu \mathrm{M}$ & $3.17 \pm 1.30$ & $0.6 \pm 0.1$ & 1.18 & $2.14 \pm 0.558$ & $0.7 \pm 0.1$ & 0.795 \\
\hline Cyclophosphamide & $\mu \mathrm{M}$ & $2571 \pm 394$ & $1.6 \pm 0.1$ & 1.52 & $1414 \pm 135$ & $1.1 \pm 0.1$ & 0.837 \\
\hline \multicolumn{8}{|l|}{ Others } \\
\hline Actinomycin D & nм & $4.20 \pm 1.21$ & $0.5 \pm 0.1$ & 15762 & $0.0488 \pm 0.0186$ & $0.5 \pm 0.1$ & 183 \\
\hline Etoposide & $\mu \mathrm{M}$ & $2.14 \pm 0.331$ & $0.6 \pm 0.1$ & 9.18 & $0.228 \pm 0.0716$ & $0.5 \pm 0.1$ & 0.975 \\
\hline Irinotecan & $\mu \mathrm{M}$ & $1.87 \pm 1.70$ & $0.6 \pm 0.1$ & 1.02 & $0.752 \pm 0.529$ & $0.7 \pm 0.2$ & 0.413 \\
\hline SN-38 & nм & $17.1 \pm 8.11$ & $0.5 \pm 0.1$ & 0.569 & $6.44 \pm 3.17$ & $0.6 \pm 0.0$ & 0.214 \\
\hline Mitomycin C & $\mu \mathrm{M}$ & $0.116 \pm 0.0265$ & $0.6 \pm 0.0$ & 5.55 & $0.131 \pm 0.0129$ & $0.7 \pm 0.0$ & 6.28 \\
\hline Tamoxifen & $\mu \mathrm{M}$ & $7.71 \pm 4.33$ & $7.5 \pm 4.0$ & 0.874 & $6.03 \pm 2.22$ & $6.6 \pm 2.4$ & 0.684 \\
\hline
\end{tabular}

The $\mathrm{IC}_{50}$ and $\gamma$ values represent the mean \pm S.D. of quadruplicate experiments. a) The relative resistance $=\mathrm{IC}_{50}$ values in Hvr100-6 cells $/ \mathrm{IC}_{50}$ values in HeLa cells.

were in the order of VDS (3.42), VCR (4.04), VLB (5.23), and NVB (5.73), indicating no significant correlation between $\mathrm{C} \log \mathrm{P}$, i.e., hydrophobicity and relative resistance. Despite a large number of investigations of MDR1, qualitative structure-activity relationship analysis has not identified the specific feature(s) of the chemical structure important for transport activity. However, empirical data have suggested that the hydrophobicity, planar structure, and/or amphipathicicity (or slight cationic character) may be important. ${ }^{32,33)}$ These results strongly suggest the necessity to determine experimentally whether anticancer drugs are substrates of MDR1.

Hvr100-6 cells also showed a high level of resistance to ACT-D and VP-16, which was reversed by CyA. Unexpectedly, Hvr100-6 cells showed resistance to 6-MP (8-fold higher than HeLa cells), which was not affected by CyA. Exposure to VLB might result in a lack of the salvage pathway enzyme hypoxanthine-guanine phosphoribosyltransferase, which is known to be involved in the mechanism of resistance to 6-MP. ${ }^{34)}$ The relative level of resistance of Hvr100-6 cells to MMC, which was reported to be a substrate of MDR1, ${ }^{35)}$ was 9 -fold compared with that of HeLa cells, and this was also almost unaffected by CyA. The present results suggest that the resistance of Hvr100-6 cells to 6-MP and MMC was not related to MDR1.

Interestingly, the $\gamma$ values in Hvr100-6 cells were similar to those in HeLa cells for all anticancer drugs, except for DXR, DNR, 6-MP, and ACT-D. The $\gamma$ values of DXR and DNR were decreased in Hvr100-6 cells, suggesting a decrease in the response to these drugs. It was reported that 


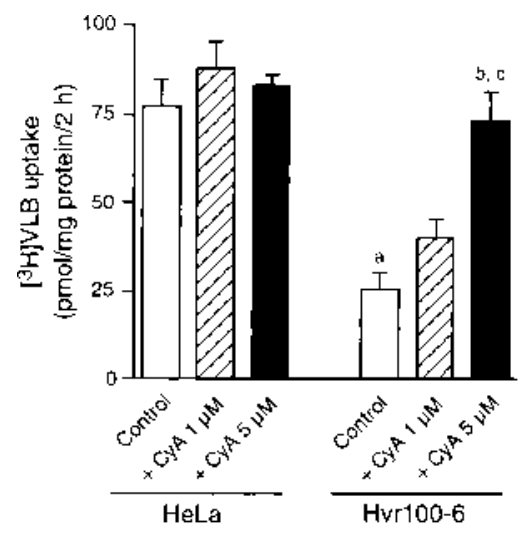

Fig. 4. Uptake of $\left[{ }^{3} \mathrm{H}\right]$ Vinblastine by HeLa and Hvr100-6 Cells in the Presence or Absence of Cyclosporin A

Cells were incubated in HBSS containing $\left[{ }^{3} \mathrm{H}\right] \mathrm{VLB} 1 \mu \mathrm{M}(37 \mathrm{kBq})$ for $2 \mathrm{~h}$ at $37^{\circ} \mathrm{C}$. $\square$, Control; $⿴ 囗 1$, with $1 \mu \mathrm{M} \mathrm{CyA;} \mathbf{\square}$, with $5 \mu \mathrm{M}$ CyA. a) $p<0.0001$ significantly different from the value of HeLa cells. b) $p<0.0001$ significantly different from the value of Hvr100-6 cells in the absence of CyA. c) $p<0.0001$ significantly different from the value of Hvr100-6 cells in the presence of CyA $1 \mu \mathrm{M}$. Each bar represents the mean \pm S.D. of quadruplicate experiments.

MDR1-expressing human leukemia cells, i.e., DXR-selected CEM-CCRF and VCR-selected K562 cells, were less sensitive to the caspase-dependent apoptoic pathway mediated by cytotoxic drugs and ligation of Fas than the non-MDR1-expressing cell lines CEM-CCRF and K562, respectively. ${ }^{36,37)}$ In contrast, the $\gamma$ values of 6-MP and ACT-D increased in Hvr100-6 cells, suggesting increases in the response to 6-MP or ACT-D, but the reasons for these observations remain unclear. Thus the alteration in $\gamma$ values suggested that MDR1 may be linked with some cellular signaling event or cell death pathway. The uptake of $\left[{ }^{3} \mathrm{H}\right] \mathrm{VLB}$ in Hvr100-6 cells was about 3-fold lower than that in HeLa cells, and this was increased by $\mathrm{CyA}$ in a concentration-dependent manner to a level comparable to that in HeLa cells (Fig. 4). These observations regarding VLB uptake in Hvr100-6 cells can explain qualitatively, but not quantitatively, the alterations in its cytotoxicity. The difference in magnitude could be attributable to a nonspecific accumulation of lipophilic VLB into the plasma membrane of the cells.

The effects of CyA on cytotoxicity suggested that the efficacy of chemotherapy could be improved by coadministration with other drugs that are substrates of MDR $1{ }^{38,39)}$ Standard regimens of combination chemotherapy have been used clinically; e.g., TXL plus CDDP for ovarian cancer, TXT plus DXR or CPA for breast cancer, CDDP plus NVB or TXL or GEM or CPT-11 for non-small cell lung cancer, and 5-FU plus leucovorin for colorectal cancer. In regimens consisting of combinations of two or more substrates of MDR1, synergistic potentiation might be achieved via an MDR1-dependent mechanism, although there is as yet no direct evidence to support this suggestion.

In conclusion, the 27 anticancer drugs examined here could be classified as substrates or nonsubstrates of MDR1 using HeLa and drug-resistant derivative cell lines. These drug-resistant derivatives of HeLa cells were newly established by stepwise exposure to VLB and successively overexpressed only MDR1, with no alterations in the expression of MRP1, MRP2, and BCRP, and therefore provided useful information for the development of effective chemotherapeutic regimens.
Acknowledgments We are grateful to each of the companies that provided the anticancer drugs used in this study.

\section{REFERENCES}

1) Kaufman D., Chabner B. A., "Cancer Chemotherapy and Biotherapy," second edition, ed. by Chabner B. A., Longo D. L., Lippincot-Raven, Philadelphia, 1996, pp. 1-17.

2) Goldstein L. J., Eur. J. Cancer, 32A, 1039-1050 (1996).

3) Marie J. P., Zhou D. C., Gurbuxani S., Legrand O., Zittoun R., Eur. J. Cancer, 32A, $1034-1038$ (1996).

4) Trock B. J., Leonessa F., Clarke R., J. Natl. Cancer Inst., 89, 917-931 (1997).

5) Leith C., Curr. Opin. Hematol., 5, 287-291 (1998).

6) Szakacs G., Jakab K., Antal F., Sarkadi B., Pathol. Oncol. Res., 4, 251-257 (1998).

7) Huet J., Laval F., Cancer Res., 45, 987-991 (1985).

8) Young I., Young G. J., Wiley J. S., van der Weyden M. B., Eur. J. Cancer Clin. Oncol., 21, 1077-1082 (1985).

9) Kobayashi T., Kakihara T., Uchiyama M., Fukuda T., Kishi K., Shibata A., Leuk. Lymphoma, 15, 503-505 (1994).

10) Obata T., Endo Y., Tanaka M., Matsuda A., Sasaki T., Cancer Lett., 123, 53-61 (1998).

11) Takano H., Kohno K., Matsuo K., Matsuda T., Kuwano M., Anticancer Drugs, 3, 323-330 (1992).

12) Copur S., Aiba K., Drake J. C., Allegra C. J., Chu E., Biochem. Pharmacol., 49, 1419-1426 (1995).

13) Taniguchi K., Kohno K., Kawanami K., Wada M., Kanematsu T., Kuwano M., Cancer Res., 56, 2348-2354 (1996).

14) Miyaki M., Ono T., Hori S., Umezawa H., Cancer Res., 35, 2015 2019 (1975).

15) Kondo Y., Woo E. S., Michalska A. E., Choo K. H., Lazo J. S., Cancer Res., 55, 2021-2023 (1995).

16) Yao K. S., Godwin A. K., Johnson S. W., Ozols R. F., O’Dwyer P. J., Hamilton T. C., Cancer Res., 55, $4367-4374$ (1995).

17) Kuo M. T., Bao J. J., Curley S. A., Ikeguchi M., Johnston D. A., Ishikawa T., Cancer Res., 56, 3642-3644 (1996).

18) Shen D., Pastan I., Gottesman M. M., Cancer Res., 58, 268-275 (1998).

19) Gottesman M. M., Pastan I., Annu. Rev. Biochem., 62, 385-427 (1993).

20) Stein W. D., Physiol. Rev., 77, 545-590 (1997).

21) Aszalos A., Ross D. D., Anticancer Res., 18, 2937-2944 (1998).

22) Loe D. W., Deeley R. G., Cole S. P., Eur. J. Cancer, 32A, 945-957 (1996).

23) Cole S. P. C., Deely R. G., Bioessays, 20, 931-940 (1998).

24) Slater L. M., Sweet P., Stupecky M., Gupta S., J. Clin. Invest., 77, 1405-1408 (1986).

25) Saeki T., Ueda K., Tanigawara Y., Hori R., Komano T., J. Biol. Chem., 268, 6077-6080 (1993).

26) Mosmann T., J. Immunol. Methods, 65, 55-63 (1983).

27) Ishiyama M., Mizoguchi M., Shiga M., Kasamoto K., Chem. Pharm. Bull., 41, 1118-1122 (1993).

28) Iida N., Takara K., Ohmoto N., Nakamura T., Kimura T., Wada A., Hirai M., Sakaeda T., Okumura K., Biol. Pharm. Bull., 24, 10321036 (2001).

29) Scheffer G. L., Kool M., Heijn M., de Haas M., Pijnenborg A. C., Wijnholds J., van Helvoort A., de Jong M. C., Hooijberg J. H., Mol C. A., van der Linden M., de Vree J. M., van der Valk P., Elferink R. P., Borst P., Scheper R. J., Cancer Res., 60, 5269-5277 (2000).

30) Kanzaki A., Toi M., Nakayama K., Bando H., Mutoh M., Uchida T., Fukumoto M., Takebayashi Y., Jpn. J. Cancer Res., 92, 452-458 (2001).

31) Takara K., Tsujimoto M., Ohnishi N., Yokoyama T., Biochem. Biophys. Res. Commun., 292, 190-194 (2002).

32) Oude Elferink R. P. J., Meijer D. K. F., Kuipers F., Jansen P. L. M., Groen A. K., Groothuis G. M. M., Biochim. Biophys. Acta, 1241, 215-268 (1995).

33) Ford J. M., Eur. J. Cancer, 32A, 991-1001 (1996).

34) Pieters R., Huismans D. R., Loonen A. H., Peters G. J., Hahlen K., van der Does-van den Berg A., van Wering E. R., Veerman A. J., Int. J. Cancer, 51, 213-217 (1992).

35) Hunter J., Hirst B. H., Adv. Drug Deliv. Rev., 25, 129-157 (1997). 
36) Smyth M. J., Krasovskis E., Sutton V. R., Johnstone R. W., Proc. Natl. Acad. Sci. U.S.A., 95, 7024-7029 (1998).

37) Johnstone R. W., Ruefli A. A., Tainton K. M., Smyth M. J., Leuk. Lymphoma, 38, 1-11 (2000).

38) Kusunoki N., Takara K., Tanigawara Y., Yamauchi A., Ueda K., Ko- mada F., Ku Y., Kuroda Y., Saitoh Y., Okumura K., Jpn. J. Cancer Res., 89, 1220-1228 (1998).

39) Takara K., Tanigawara Y., Komada F., Nishiguchi K., Sakaeda T., Okumura K., Biol. Pharm. Bull., 22, 1355-1359 (1999). 